\title{
The Hall Technique: an educational tool
}

\section{By Lucy Malcolm \& Lauren Pengilley}

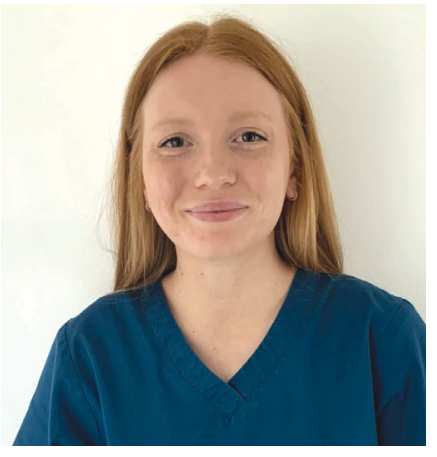

Lucy Malcolm

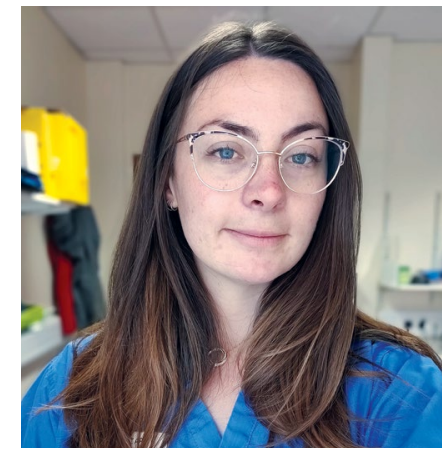

Lauren Pengilley

During our time in Inverness as dental core trainees we designed and produced a ten-minute educational video focusing on the Hall Technique, winning first prize at the West of Scotland BDA and FGDP Postgraduate Presentation Evening.

We were inspired to produce this virtual resource for a variety of reasons. As undergraduate dental students we were taught the theory and practical elements of the Hall Technique, and had opportunity to practise in outreach clinics to gain competency. This was a technique we felt confident carrying out, yet during the research phase of this project we found a recent $\mathrm{CPD}$ event highlighted a lack of confidence

amongst GDPs in using the technique and limited available online resources detailing the method.

Another reason grew from the COVID-19 pandemic. During the last year COVID-19 prompted us to consider aerosol generating procedures (AGPs) and how we best manage them within day to day practice. With the use of separators, the Hall Technique avoids the need for an AGP so removes fallow time and donning of excessive PPE - which may be frightening to a child. This treatment modality may also be carried out by dental therapists and as we move out of the pandemic, shared care will be more important than ever to overcome the backlog of delayed treatments.

Moreover, COVID-19 has had a huge impact on dental education with a significant shift to remote and online learning. This was yet another reason to warrant the production of a comprehensive online learning tool for students and DCPs alike.

After considering the above we began our project, aiming to develop a resource which would reflect the current situation in dentistry.

During production we utilised a variety of information sources, referenced in the video, to discuss the effectiveness of the Hall Technique. The video was structured to contain: indications and contraindications; phantom head simulation; and a live patient demonstration. NHS Highland Medical Illustration and Data Protection were consulted to ensure appropriate consent was gained. 
4 Following on from production we presented to secondary care staff, then 40 dental therapy students at an annual conference to acquire early feedback from a range of perspectives. Formal feedback received was overwhelmingly positive, indicating the video was set at an appropriate level for students and professionals at different stages in their career.

We concluded that the final educational video had successfully met our aim and had shown that online educational tools are a valuable source of education. Entering the BDA and FGDP Presentation Evening showcased our work to a wider audience and we were both delighted and shocked to win first prize!

Moving forward, we aim to create similar resources which we hope prove valuable to undergraduate students and qualified DCPs within the wider dental community.

The video can be viewed at the following link (the live patient demonstration has been removed from this version): https://stream.uht ac.uk/Player/I8ed8f6D.

\section{An exciting new partnership}

The College of General Dentistry and the National Health Service Retirement Fellowship are forming a new collaboration that will benefit their individual members and patients across the UK.

The NHS Retirement Fellowship, a registered charity, offers friendship, fellowship and fun to retired NHS and social care staff and their partners, providing a bridge between life in employment and new opportunities in retirement. With branches throughout England, Scotland and Wales its 7,000 plus members can meet and enjoy a variety of social, leisure and educational events such as walking, golf, photography and sightseeing. It provides access to a great range of discounts and benefits, including travel, annual worldwide travel, home and car insurance, shopping discounts and members can become a member of the Civil Service Club in Whitehall, London which offers overnight accommodation, a bar and more formal dining area.

The new partnership blends the benefits of professional, personal and social interaction with old and new friends and colleagues. The College is keen to encourage former longstanding registrants of the GDC to maintain membership of the College, possibly forming an informal Forum or Guild, within the College. Retired colleagues have a wealth of talent that the College would not want to lose. This is one of the areas in which the College and the NHS Retirement Fellowship will be forging a strong link, with proposals for retired dental professionals to enjoy the benefits of both organisations.

Another important benefit is the experience of retired health and social care colleagues as knowledgeable patients. Colleagues from the Fellowship will work with the College to ensure information for patients and the public is accessible and reflects what patients need and expect from dental professionals in the twenty-first century. Together the two organisations will ensure that College publications are patient-friendly and free of jargon. The College will be seeking to develop a robust public engagement mechanism, with the help and support of Fellowship members, to build public trust and confidence in dentistry.

College of General Dentistry: https://cgdent.uk

NHS Retirement Fellowship: https://nhsrf.org.uk 